\title{
Combination Therapy Using Nonablative Fractional Laser and Intralesional Triamcinolone Injection for Hypertrophic Scars and Keloids Treatment
}

\author{
Sung-No Jung, Jin Tae Cho, Jongweon Shin \\ Department of Plastic and Reconstructive Surgery, Uijeongbu St. Mary's Hospital, \\ College of Medicine, The Catholic University of Korea
}

\section{Introduction}

Hypertrophic scars and keloids are benign hyperproliferative growths of dermal collagen. Patients with these conditions often experience physical problems as well as psychological concerns. No single modality is universally effective and combinations of treatment modalities are proved to be more effective than monotherapy, we sought to elicit the clinical efficacy of combination therapy using intralesional injection (ILI) and nonabl ative fractional LASER (NAFL) compared to ILI therapy alone.

\section{Materials and Methods}

Total of 38 patients with hypertrophic scars or keloids were enrolled. 21 patients who received ILI alone (control group) and the other (17 patients) who received NAFL plus ILI simultaneously (combined group) were evaluated. ILI was conducted with following manner: 1:1 mixture of triamcinolone $(40 \mathrm{mg} / \mathrm{mL})$ and lidocaine $2 \%(400 \mathrm{mg} / 20 \mathrm{~mL})$ was injected. For the NAFL, a $1550 \mathrm{~nm}$ fractional erbium-glass laser was utilized. To evaluate the results of each group, Patient and Observer Scar Assessment Scale (POSAS) was used.

\section{Results}

Patients' demographics are summarized in table 1. The POSAS was divided into three categories and evaluated; patient's scale (PS), observer's scale (OS) and total score (PS+OS). There was no initial difference on both OS and PS, but the score showed a significant discrepancy at 6 months (Table 2). The recurrence rate was calculated and there was no significant difference (Table 3).

\section{Discussion}

Simultaneously combining NAFL to traditional ILI in treatment of hypertrophic scars and keloids can reduce treatment sessions to fewer numbers. It is more effective in symptoms care and subjective contentment improvement with longer symptoms free duration, although it could not lower the recurrence rate. Hence, combination therapy with NAFL and ILI may be considered as a reasonable therapeutic option for hypertrophic scars and keloids.

Table 1. Summary of patients' demographics.

\begin{tabular}{|l|l|l|}
\hline & Control group & Combined group \\
\hline Number of patients (Male:Female) & 21 (8:13) & 17 (9:8) \\
\hline Mean age (years) & 41.38 (range 18-62) & 37.23 (range 19-62) \\
\hline Type of scar & & \\
Hypertrophic scar & 12 & 8 \\
Keloid & 9 & 9 \\
\hline Lesion age (months) & 15.38 (range 6-23) & 16.76 (range 6-24) \\
\hline Location & 2 & \\
Head \& neck & 2 & 3 \\
Trunk & 9 & 8 \\
Upper extremity & 2 & 2 \\
Lower extremity & 3 & 1 \\
Joint & 5 & 3 \\
\hline Etiology & & 8 \\
Trauma & 9 & 9 \\
Surgery & 12 & \\
\hline
\end{tabular}

Table 2. Summary of the results of the number of treatment sessions and POSAS. (D-: difference between initial and after 6 months scale scores)

\begin{tabular}{|l|l|l|l|}
\hline & Control group & Combined group & P value \\
\hline Number of treatment sessions & 6.95 & 5.47 & 0.042 \\
\hline Patient's scale (PS) & & & \\
$\quad$ Initial & 38.38 & 36.18 & 0.580 \\
$\quad$ After 6 months & 23.77 & 13.36 & 0.004 \\
\hline Observer's scale (OS) & & & \\
$\quad$ Initial & 42.08 & 38.18 & 0.302 \\
$\quad$ After 6 months & 24.15 & 17.64 & 0.046 \\
\hline PS+OS & & & \\
$\quad$ Initial & 80.46 & 74.36 & 0.347 \\
$\quad$ ffter 6 months & 47.92 & 31.00 & 0.002 \\
\hline D-PS & 14.62 & 22.82 & 0.005 \\
\hline D-OS & 17.92 & 20.55 & 0.549 \\
\hline D-(PS+OS) & 32.54 & 43.36 & 0.041 \\
\hline
\end{tabular}

Table 3. Recurrence rate and remission period in each group

\begin{tabular}{|l|l|l|l|}
\hline & Control group & Combined group & P value \\
\hline Recurrence rate & $38.1 \%(8 / 21)$ & $35.3 \%(6 / 17)$ & 0.859 \\
\hline Remission period (months) & 3.00 & 4.17 & 0.042 \\
\hline
\end{tabular}

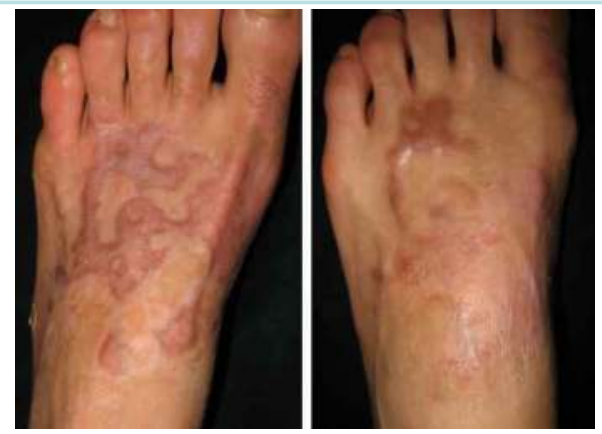

Figure 1. Hypertrophic scar in the foot dorsum developed after burn injury (left) and the same scar after 6 months from the last session of combination therapy (right). The tightness and itching sensation were resolved and the overall quality of the scar was much improved

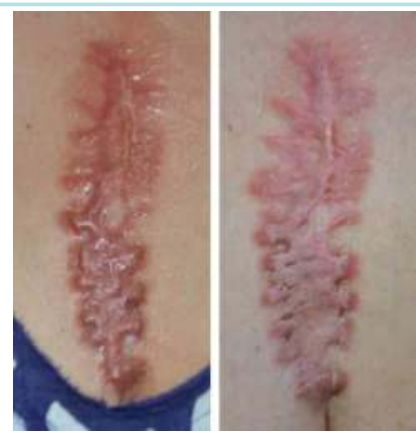

Figure 2. Keloid scar in the chest wall occurred after surgery (left). 6 sessions of combination therapy were applied and the treatment was stopped. After months, subjective symptoms and assessment was much improved while objective advancement was disappointing (right). 